\title{
A comparison of two response procedures for reaction time experiments
}

\author{
STEVEN E. POLTROCK \\ University of Washington, Seattle, Washington 98195
}

\begin{abstract}
Response procedures in binary-classification reaction time experiments typically require that one of two keys or buttons be depressed, either with one finger used for both keys or with separate fingers used for each key. An experimental comparison of the one-finger and two-finger response procedures indicated that the variance in reaction time for one-finger responses was more than twice the variance for two-finger responses. It was concluded that the two-finger response procedure permits more sensitive experimental designs.
\end{abstract}

Reaction time (RT) is a commonly used dependent variable in experimental investigations of perceptual and cognitive processes. To maximize the sensitivity of an RT experiment, a procedure should be chosen which minimizes those components of RT variance not due to the experimental manipulation. One factor which may be expected to influence RT variance is the response procedure.

The response procedures most commonly used in binary-classification RT experiments require that the subject indicate the result of a decision by pressing one of two buttons or keys. Some experimenters (e.g.. Cohen. 1972; Cooper. 1975; Kristofferson, 1972; Posner \& Boies. 1971) have required that the subject rest the index finger of each hand on a key or button, which is depressed with the appropriate finger for a response. Other experimenters (e.g., Corballis \& Miller. 1973; Juola, Fischler, Wood, \& Atkinson, 1971: Klatzky, 1972; Wescourt \& Atkinson, 1973) have required that the subject use only one index finger. which rests at a central location, and respond by depressing a key or button located to the right or the left.

Typically, no rationale is presented for using one procedure vs. the other. This paper takes the view that. other things being equal, the procedure that yields the least error variance should be selected. The present experiment was designed to compare the error variance associated with the two response procedures.

\section{METHOD}

\begin{abstract}
Subjects
The subjects were 32 undergraduate students at the University of Washington who participated for extra credit in an introductory
\end{abstract}

This research was supported by the National Institute of Mental Health, Grant MH 21795, to the University of Washington (Earl Hunt, principal investigator). The author wishes to thank Geoffrey Loftus, Tom Nelson, and Ben McKeever for their critical reading of this manuscript. Geoffrey Loftus sponsors this paper and takes full editorial responsibility for its content. psychology course. Half the subjects were instructed to respond with one finger, and half were instructed to respond with two fingers.

\section{Procedure}

Subjects were instructed and tested in groups of one to four, with all members of a test group receiving the same instructions. The experiment was conducted with each subject seated in a soundproof booth. On each trial of the experiment, an asterisk was presented in the center of a computer-controlled display as a warning signal. After an interval of 400 to $600 \mathrm{msec}$, chosen randomly from a uniform distribution, a test asterisk was presented $1.9 \mathrm{~cm}$ to the left or the right of the warning asterisk. The orientation of the test asterisk was determined randomly for each trial. but was the same for all subjects in a test group. The warning asterisk and the test asterisk were displayed together for $2 \mathrm{sec}$.

Subjects were instructed to respond with a key on the right if the test asterisk appeared on the right, or a key on the left if the asterisk appeared on the left. Subjects were told to respond as quickly as possible without making errors. The instructions given to the two treatment groups differed only with respect to the mechanics of the response. Subjects using the two-finger procedure were instructed to rest an index finger on each of the response keys, and to press the appropriate key for the response. Subjects using the one-finger procedure were instructed to rest the index finger of the dominant hand on a central key, and to press a key to the right or the left of the central key for the response. Subjects were allowed to respond up to 2 sec after stimulus presentation. The response decision rule was highly compatible and was expected to lead to rapid responses and low error rates. If the subject responded correctly, his or her RT was displayed in milliseconds; otherwise, the word WRONG was displayed.

Each subject participated in three blocks of 210 trials, with a 1 -min rest between blocks. Trials were presented at the rate of 15 per minute, requiring $44 \mathrm{~min}$ to complete the experiment.

\footnotetext{
Apparatus

The presentation of stimuli and the recording of responses were under the control of a NOVA 800 computer. Stimuli were presented on Tektronix 602 display screens with P-15 phosphor. A specially designed response panel included eight hinged keys resting on microswitches. The response keys were $2.5 \mathrm{~cm}$ wide and either 7.5 or $10 \mathrm{~cm}$ in length. The keys were aligned in parallel with $.5 \mathrm{~cm}$ between adjacent keys. The keys used for two-finger responses were $10 \mathrm{~cm}$ in length and were the leftmost and rightmost keys on the panel. The $7.5-\mathrm{cm}$ keys were used for one-finger responses. The subject rested an index finger on a central key and responded with the adjacent keys. With either response method, the subjects' fingers were directly over the microswitch so that key length would not affect $\mathrm{RT}$.
} 


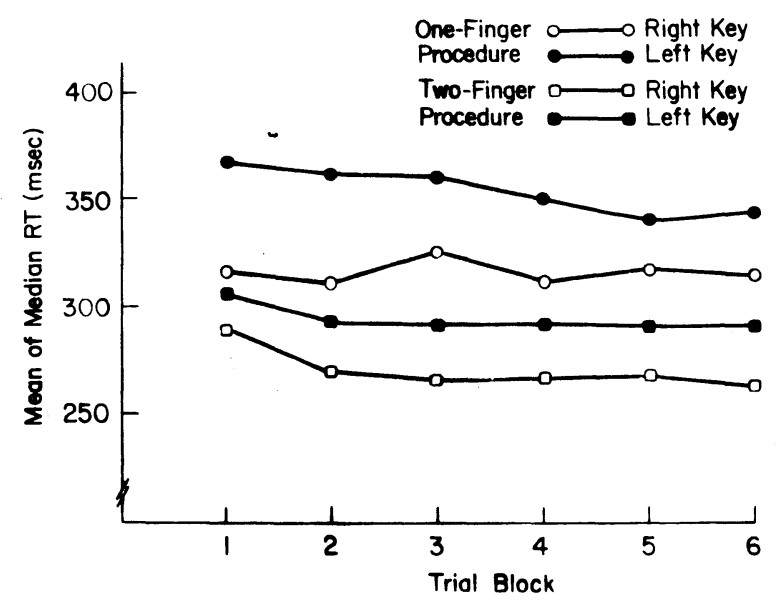

Figure 1. Mean of median reaction time (RT) for the one-finger and two-finger response procedures as a function of trial block.

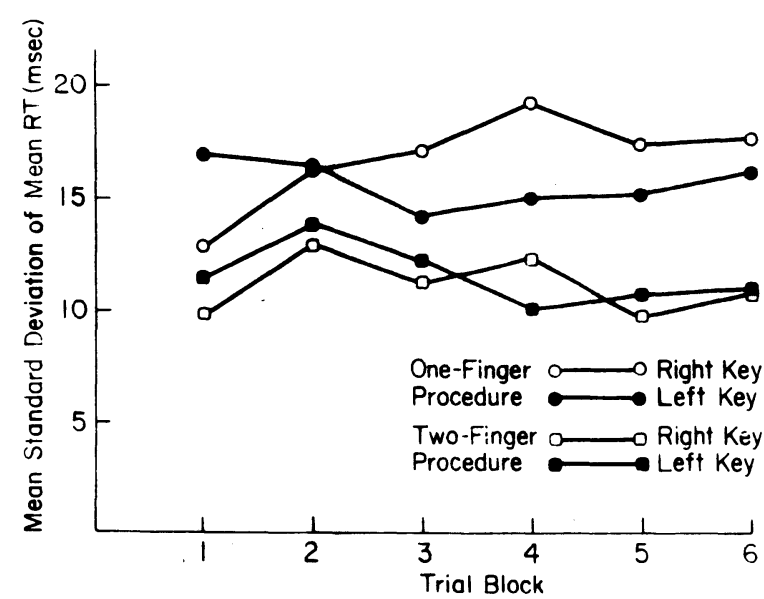

Figure 2. Mean standard deviation of mean reaction time (RT) for the one-finger and two-finger response procedures as a function of trial block.

\section{RESULTS}

Subjects' responses on the first 30 trials were discarded as practice trials. The remaining 600 trials were divided into 100 trial blocks to permit analyses of changes in performance during the experimental session. The means of the median RTs for correct left and right responses produced with both response procedures are presented in Figure 1 as a function of 100 trial blocks. The median response times are based on approximately 50 observations for each key pressed by each subject. Responses are significantly faster for the two-finger procedure, $F(1,30)=12.91, p<.002$, and faster for the right key than the left, $F(1,30)=19.20, p<.001$. A significant effect of trial blocks, $F(5,150)=4.31, \mathrm{p}<.001$, is due to a decrease in RT from Block 1 to Block 2: A comparison of Block 1 with the other five blocks was highly significant, $F(1,150)=17.48, \mathrm{p}<.001$, while the residual was not significant. $F(4,150)=1.02$, $p>.1$. The interactions of response procedure with key, response procedure with trial block, key with trial block, and the three-way interaction were not signiticant, $F(1,30)=1.07, F(5,150)=1.10$, $F(5,150)=.94$, and $F(5,150)=1.95$, respectively, with $\mathrm{p}>.05$ for all tests.

The standard deviation of each subject's mean RT for correct responses was computed for each key over a block of 100 trials. These standard deviations were entered as scores in an analysis of variance. The mean standard deviation for each key and each response procedure is presented in Figure 2 as a function of trial block. The standard deviations produced using the two-finger procedure were significantly smaller than those produced with the one-finger procedure, $\mathrm{F}(1,30)=11.11, \mathrm{p}<.005$. There were no significant effects of trial block or key, $F<1$ in both cases, and none of the interactions approached significance.

An error was scored on a trial if a subject pressed the wrong key or failed to press a key within $2 \mathrm{sec}$. The mean error rate for each response procedure is presented in Table 1 as a function of trial block. The response procedure did not significantly affect the error rate, $F(1,30)=2.04, p>.1$, but errors increased significantly over trials, $F(5,150)=6.12$, $\mathrm{p}<.001$. The Response Procedure by Trial Block interaction was not significant, $F(5,150)=1.14$, $\mathrm{p}>.1$.

\section{DISCUSSION}

The purpose of this experiment was to compare the RT variance associated with the response procedures. However, other aspects of the response behavior, the error rate and the RT variance introduced by counterbalancing, could also influence an experimenter's choice of a response procedure.

Error rates in RT experiments are typically small, and results from error trials are usually not included in analyses. Errors are generally assumed to arise from encoding or decision processes, rather than the response procedure. A response procedure that led to high error rates would not be appropriate for RT studies. Both procedures led to low error rates, less than $5 \%$ of the trials, so neither may be rejected on this basis.

Experimental designs often require counterbalancing of the binary classification over keys, so that half the subjects use the right key for one value of a classification, while the other subjects use the left key. Differences in RT for the two keys increase the RT variance for the classification. However, the increase is not included in the error variance if an analysis of variance is performed with counterbalancing treated as a between-subjects variable. If the analysis to be performed does not account for the variance due to counterbalancing, then a response procedure should be chosen to minimize the difference in RT for the two keys. Since the Response Procedure by Key

Table 1

Mean Proportion of Trials on Which an Error Occurred

Response Procedure

Trial Block One Finger Two Fingers

\begin{tabular}{ccc}
\hline 1 & .015 & .015 \\
2 & .021 & .026 \\
3 & .014 & .034 \\
4 & .028 & .048 \\
5 & .029 & .055 \\
6 & .035 & .060 \\
Mean & .024 & .040 \\
\hline
\end{tabular}


interaction was nonsignificant for median $\mathrm{RT}$, neither procedure can be recommended on this basis.

A factor that does discriminate between the response procedures is the RT standard deviation or variance. The mean variance of the mean RT for the one-finger procedure, $261.8 \mathrm{msec}^{2}$, was more than twice the mean variance for the two-finger procedure, $127.7 \mathrm{msec}^{2}$. The features that distinguish the response procedures are the use of one hand and finger vs. two, and the lateral finger movement required only for the one-finger responses. It is logical to assume that the lateral finger movement contributes considerably to the difference between the mean variances. Of course, the variance associated with the finger movement depends on the particular response apparatus used, but any finger movement can be expected to introduce some variance. Therefore, the two-finger procedure permits a more sensitive test of any hypothesis involving processing stages that are presumed to be independent of the response stage. This conclusion is strengthened by the failure to find a significant effect of trial blocks on the standard deviation of the mean RT. Though practice leads to reduced RTs, it does not significantly reduce the portion of the variance due to the response procedure.

\section{REFERENCES}

Conen, G. Hemispheric differences in a letter classification task. Perception \& Psychophysics, 1972, 11, 139-142.
Cooper, L. A. Mental rotation of shapes. Cognitive Psychology, $1975,7,20-43$.

Corballis, M. C., \& Miller, A. Scanning and decision processes in recognition memory. Journal of Experimental Psychology, 1973, 98, 379-386.

Juola, J. F., Fischler, I., Wood, C. T., \& Atkinson, R. C. Recognition time for information stored in long-term memory. Perception \& Psychophysics, 1971, 10, 8-14.

KLATZKY, R. L. Visual and verbal coding of laterally presented pictures. Journal of Experimental Psychology, 1972, 96, 439-448.

KRISTOFferson, M. W. When item recognition and visual search functions are similar. Perception \& Psychophysics, 1972, 12, 379-384.

Posner, M. I., \& Bores, S. J. Components of attention. Psychological Review, 1971, 78, 391-408.

Wescourt, K. T., \& AtKanson, R. C. Scanning for information in long- and short-term memory. Journal of Experimental Psychology, 1973, 98, 95-101. 